\title{
Mono- and polysynaptic reflexes of the trigeminal muscles in human beings
}

\author{
H.-J. HUFSCHMIDT AND H. SPULER \\ From the Department of Neurology and Neurosurgery of the University \\ of Würzburg, Germany
}

The afferent nerves of the trigeminal musculature run in different anatomical tracts. The Ia fibres from the muscle spindles run in the motor root and reach the medulla without suffering interruption of any kind. From the studies of Szentagothai (1948) it was learned that the cells of the Ia fibres which correspond to the spinal ganglion cells lie in the region of the mesencephalic root of the fifth nerve: these are the large afferents from muscle spindles. The Ib fibres of the Golgi apparatus run with the other afferent fibres of the trigeminal muscle which form the sensory root and have their origin in the cells of the Gasserian ganglion. The electrophysiological studies of McIntyre (1951) proved that the monosynaptic reflex from the muscle spindles runs through the mesencephalic root of the trigeminus. Because of the different anatomical tracts of the Ia and Ib fibres, it is possible to separate the mono- from the polysynaptic influences by cutting the sensory root, as is done in the Frazier-Spiller operation often employed in severe cases of trigeminal neuralgia. Again, in cases with damage to the dorsal mesencephalic region it is to be expected that on the damaged side the monosynaptic reflex might be absent. The pathological situation as well as surgical intervention make it possible then to separate the monosynaptic from the polysynaptic reflexes; to throw light on the question of the silent period that follows a monosynaptic reflex; and to associate the inhibition of the activity which occurs during excitation of a muscle with definite afferent fibres. It is of particular interest to find out what happens to the silent period when the sensory root has been severed surgically as only the Ia fibres and the axons of the motoneurones remain.

\section{METHODS}

The monosynaptic reflex is easily obtained by a sharp blow to the mandible. It is possible to study the mechanical effects of the masseter being stretched by comparing results obtained with the mouth closed or opened respectively. In the former case, the blow upon the mandible leads to an increase in tension without much stretch of the muscle, whereas in the latter the muscle is simultaneously stretched.

The masseter was electrically stimulated with the cathode placed on the muscle belly while the anode was placed over a distant extremity. The intensity of the current was increased until a muscle contraction was obtained. Thereafter, the trigeminal musculature was always inhibited for a period of 60 to $80 \mathrm{~m} . \mathrm{sec}$.

We observed 10 normal patients, four patients with a clinically demonstrable unilateral mesencephalic disease, and four patients recovering after a Frazier-Spiller operation, as well as two patients in whom the Gasserian ganglion had been infiltrated with alcohol.

The action potentials were recorded by means of insulated or naked needle-electrodes in a four-channel cathode ray-oscillograph. For electrical excitation a thyratrone stimulator was used which delivered a square wave shock of short duration ( 2 to $3 \mathrm{~m}$.sec.). On increasing the strength of the stimulus nothing could be seen in the electromyogram unless there was a simultaneous contraction.

\section{RESULTS}

The monosynaptic reflex of the masseter was usually obtained by a blow upon the mandible, the patient keeping the mouth partly opened. This produces a brief stretch and an increase in tension of the muscle. The monosynaptic reflex is followed by a silent period of about $60 \mathrm{~m} . \mathrm{sec}$. According to the studies of Struppler, Kessel, and Weidenbach (1960) the same is seen in the temporal muscles (Fig. 1).

If the patients keep the jaw tightly closed a blow upon the mandible leads to an inhibition of the electrical activity without a previous monosynaptic reflex. The duration of this inhibition corresponds to the duration of the silent period of a monosynaptic reflex. By this procedure, stretching the masticatory muscles is avoided, or is at least, small, and there is chiefly an increase of tension. Therefore, it is very probable that the tension receptors or Golgi apparatus of the masticatory musculature are responsible for the inhibition seen under such circumstances (Fig. 2). 


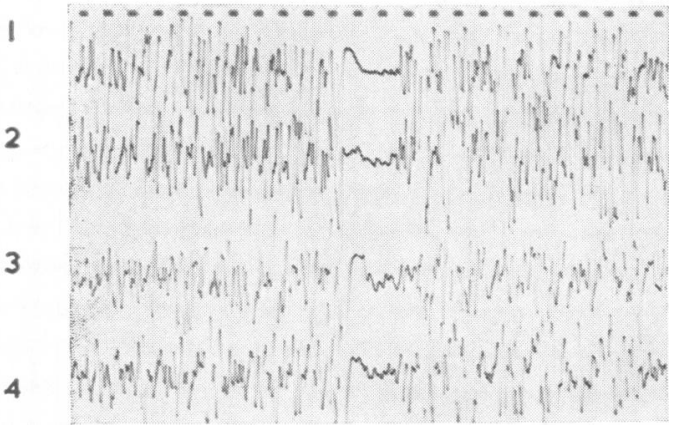

FIG. 1. Demonstration of a monosynaptic reflex of the masticatory musculature. First and second leads: right and left masseter; third and fourth: right and left temporalis. $A$ blow on the opened lower jaw produced a monosynatpic discharge followed by a silent period in all four muscles. Time: $50 \mathrm{~Hz}$.
A

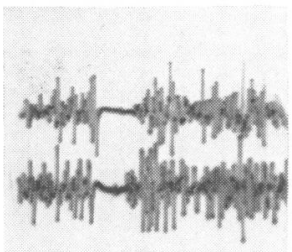

B

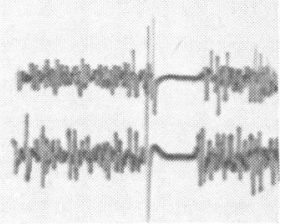

FIG. 2. A Increase in tension in masseter with autogenetic inhibition following; $\mathrm{B}$ increase in tension and length in masseter followed by reflex and autogenetic inhibition.

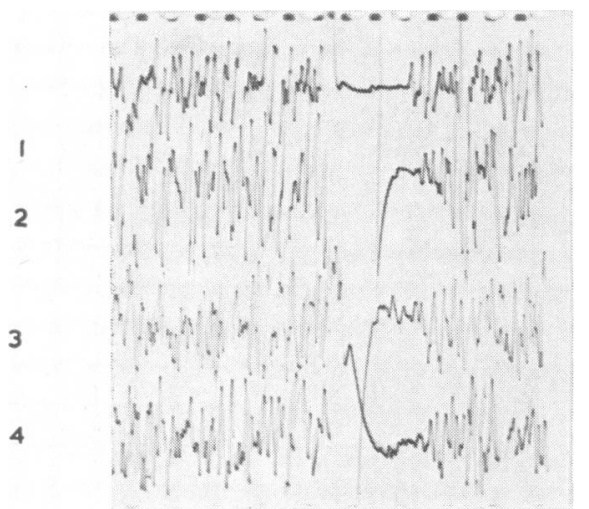

FIG. 3. Direct electric stimulation of the left masseter (second lead). The contraction of the left masseter is followed by an inhibition of the same muscle which lasted $60 \mathrm{~m} . \mathrm{sec}$. as well as of the right masseter (first lead) and both temporalis muscles (third and fourth leads). Time: $\mathrm{Hz}$.
By a direct electrical stimulation of one masseter the innervation of this muscle is inhibited for about $60 \mathrm{~m} . \mathrm{sec}$. The value corresponds, as in case 1 , to the silent period following a monosynaptic reflex. If we record at the same time the action potentials of both masseters and both temporal muscles, a synchronous inhibition is seen in all the four muscles (Fig. 3). The results permit the conclusion that in this case the tension receptors of a muscle are able to inhibit the motor neurones of synergic muscles even when they are contralateral.

After a unilateral Frazier-Spiller operation, i.e., after section of the sensory root, the silent period after the monosynaptic reflex is absent on the operated side (Fig. 4). This is again in agreement with the results of Struppler et al. (1960). The supposition that the silent period following a monosynaptic reflex is an inhibitory reflex caused by the stimulation of the tension receptors is thereby confirmed. Only occasionally a monosynaptic reflex is obtained on the operated side. The absence of this reflex is due to the fact that usually during the surgical procedure the sensory Ia fibres of the motor root are severed.

In 1948 Szentagothai discovered that after damage to the mesencephalic tracts of the trigeminal muscles, the synaptic knobs of the motor nucleus of the trigeminal muscles degenerate and McIntyre (1951) demonstrated electrophysiologically that the monosynaptic impulses run in the mesencephalic tracts. It was therefore of interest to study the pathological cases that presented a unilateral lesion of the mesencephalic regions. As expected, we could observe unilateral failure of the monosynaptic reflex while the inhibitory effect was still present on both sides (Fig. 5). Just as in animal experiments, we can prove in human pathological cases that the monosynaptic reflex runs in a special tract and that the subsequent silent period does not depend upon its presence or absence but is elicited by stimulation of different muscle receptors sensitive to tension.

Direct stimulation of the masseter proved to be of considerable interest. In normal patients, the stimulation of one masseter leads to an inhibition in the activity of both sides. In operated patients (Frazier-Spiller operation) stimulation of the operated side is without effect whereas stimulation on the unoperated side leads to a sudden inhibition of the contralateral masseter (Figs. 6a and 6b). Owing to the large stimulus artefact because of the proximity of the stimulating and recording electrodes, the precise beginning of the silent period in the stimulated side was not evident. The important point, however, lies in the fact that from the operated side it is impossible to inhibit the contralateral side. We must conclude, therefore, that the tension receptors of the operated side are unable to conduct the 


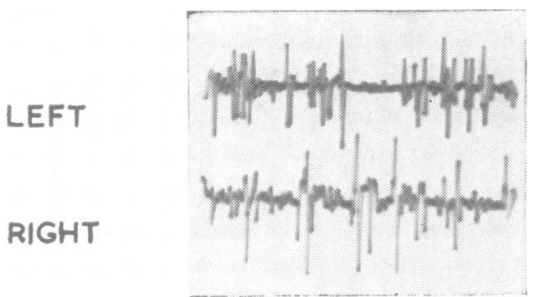

FIG. 4. Frazier-Spiller operation on the right side. Autogenetic inhibition appears only on the left side and as a consequence of an increase of tension.

A

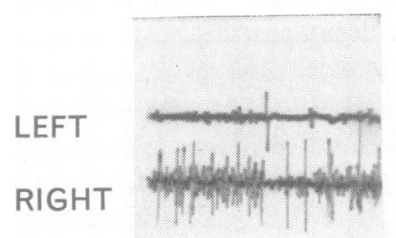

FIG. 5. Mesencephalic pathological process (tumour) with absence of monosynaptic reflex on the side of the lesion. The behaviour of the mechanism of inhibition is demonstrated on both sides. A Pathological process on the right side; B pathological process on the left side.

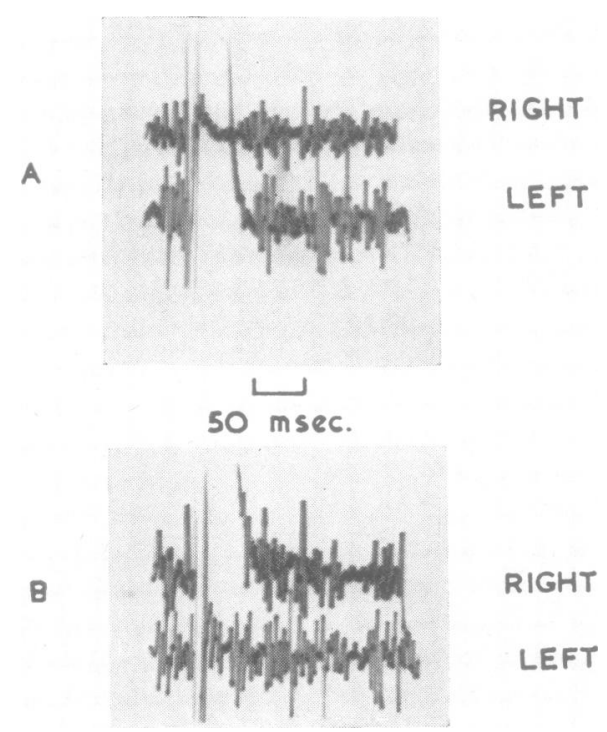

FIG. 6. Frazier-Spiller operation on the right side. A Direct muscular stimulation of the left masseter. On both sides it leads to a clear inhibition that in duration approximately corresponds to the normal value; B direct muscular stimulation of the right masseter. No inhibition to be seen. Due to unavoidable proximity of stimulating and recording electrodes, the pattern of activity of the stimulated muscle is not so easy to demonstrate but essentially it is the same as that of the opposite side. impulses to the central nervous system whereas on the unoperated side the impulses from the tension receptors can inhibit the motor neurones of the operated side.

\section{DISCUSSION}

The monosynaptic reflex of a muscle is evoked by a brief stretch stimulus. The monosynaptic reflex is followed by a silent period, as first demonstrated by Hoffmann (1920). The inhibition of the innervation was then interpreted in different ways. According to a review by Granit (1955) the silent period is composed of two peripheral factors: 1 , arrest of impulses of the muscle spindles during the contraction; 2 , the autogenetic inhibition from tension receptors and of two central factors; 3 , the recurrent inhibition, i.e., the Renshaw feedback mechanism; and 4, the subnormal phase of the motor neurones.

Matthews (1931) observed that during a muscular contraction the discharges from the muscle spindles are arrested. This suggested that the pause after a monosynaptic reflex, i.e., during a reflex contraction, could be interpreted as a cessation of the spindle impulses (Fulton and Pi-Suñer, 1928). The results of Merton (1951) confirmed this interpretation, and he believed that he could further conclude following Hoffmann's view (see Hansen and Hoffmann (1922)) that a great part of the voluntary innervation was really of a reflex nature. As our results show, there is always an inhibition, even when no previous monosynaptic reflex (or reflex contraction) can be seen. Therefore, a pure inhibition could be obtained with only an increase in muscular tension. This deduction is made not only from the present experiments, for if a muscle of a patient with arreflexia (polyneuritis) is stretched, there is in many cases an inhibition without previous reflex contraction (Hufschmidt and Schaltenbrand, 1959). Hoffmann and Jung had observed in 1937 that (by using an adequate technique for recording the monosynaptic reflex) the response to a tendon tap in patients with Adies' syndrome failed to elicit a monosynaptic reflex and only a silent period would appear. In such pathological cases the afferent tract of the monosynaptic reflex is interrupted but not the polysynaptic reflex from the Golgi tendon receptors. Finally, Fig. 4 showed that after a unilateral interruption of the polysynaptic afferent tracts in the sensory root of the trigeminal nerve, the inhibition after stretch or increase in tension disappeared on the operated side. The silent period after a monosynaptic reflex is therefore, as DennyBrown (1928) supposed, a polysynaptic inhibitory reflex. In this connexion let us refer to the study of passive muscle relaxation (Hufschmidt, 1959; 1961): 
by passive shortening of the muscle the voluntary innervation goes on normally so that voluntary contraction is not necessarily dependent on impulses from the muscle spindles as Merton (1951) affirmed.

If the masseter is caused to contract by electric stimulation, the contraction is followed by an inhibition of voluntary activity identical with the one that follows a monosynaptic reflex or an increase in tension of a muscle. Therefore it raises the question whether the inhibition might be recurrent in origin rather than be set up by the tension receptors. If this inhibition were of a recurrent origin, there would have to be recurrent collaterals from the motor axons of the trigeminus and a link between the recurrent collaterals and the contralateral side of the spinal cord.

According to Cajal (1952) the motor axons of the trigeminal nerve have no recurrent collaterals. If there were such structures, then a direct contraction of the muscle on the side with section of the sensory root should lead to an inhibition of both masseters. According to our results, this is not the case, as may be seen in Figs. $6 a$ and $6 \mathrm{~b}$ which show that from the intact side we can obtain an inhibition of both masseters whereas from the operated side no inhibition can be elicited.

Even though recurrent inhibition need not be considered in the present case, we must suppose that it initiates the silent period of the muscles of the limbs whose motor nerves have such collaterals.

The subnormal phase of the motor neurones may also contribute to the inhibition and, since the voluntary innervation goes on steadily on the operated side as well as on the unoperated side and the inhibition appears with or without a monosynaptic discharge, this factor must be taken into account.

The autogenetic muscle inhibition provides a sufficient explanation for the present results. It is very probable that the results obtained in the studies of the masticatory muscles are also valid for the motor musculature of the extremities. However, we cannot exclude that the pause in the discharge from the muscle spindles also plays a part whenever they do pause. The results presented in Fig. 5 demonstrate the identity of the human and animal organization of the trigeminal reflexes. On the side of the pathological mesencephalic processes the monosynaptic reflex fails while the polysynaptic inhibitory reflexes conducted by the sensory root remain. This also proves that the inhibition has its main source in tension receptors.

\section{SUMMARY}

In a study of the 'silent period' of contraction in the masseter muscles, advantage has been taken of the facts that tension (Golgi) and length receptors (muscle spindles) run separately in the sensory and motor roots respectively, and that surgical cases can be obtained which have undergone the FrazierSpiller operation and therefore are lacking the input from the Golgi apparatus. A comparison of normals and patients led to the following results: An increase in tension and length of the masseter muscles leads to a monosynaptic reflex followed by a silent period of the masseters as well as of the temporal muscles. Increase in tension of the masseters alone leads to a 'pure' inhibitory reflex of the masticatory musculature without monosynaptic discharge. Isolated contraction of one masseter obtained by an electric stimulation leads to inhibition of the whole masticatory musculature.

In unilateral mesencephalic diseases, the monosynaptic reflex is absent on the side of the lesion (damage to the tractus mesencephali trigemini). After surgical unilateral section of the sensory root the inhibitory polysynaptic reflexes are absent on the operated side. It is proved that the silent period after monosynaptic reflexes as well as after a direct muscular stimulation is an autogenetic inhibition from the muscle.

We are grateful for a grant from the Deutsche Forschungsgemeinschaft.

\section{REFERENCES}

Cajal, S. Ramon y (1952). Histologie du Système Nerveux, Vol. I, p. 873. Instituto Ramon y Cajal, Madrid, 1952.

Denny-Brown, D. (1928). Proc. roy. Soc. B, 103, 321.

Fulton, J. F., and Pi-Suñer, J. (1928). Amer. J. Physiol., 83, 554. Granit, R. (1955). Receptors and Sensory Perception, Yale Univ. Press, New Haven.

Hansen, K., and Hoffmann, P. (1922). Z. Biol., 75, 293.

Hoffmann, P. (1920). Ibid., 70, 515.

, and Jung, R. (1937). Schweiz. med. Wschr., 67, 566.

Hufschmidt, H.-J. (1959). Z. Biol., 111, 75.

- (1961). Pfiügers Arch. ges. Physiol., 272, 297.

, and Schaltenbrand, G. (1959). Dtsch. Z. Nervenheilk., 179, 252. McIntyre, A. K. (1951). Nature (Lond.), 168, 168.

Matthews, B. H. C. (1931). J. Physiol. (Lond.), 72, 153.

Merton, P. A. (1951). Ibid., 114, 183.

Struppler, A., Kessel, F. K., and Weidenbach, W. (1960). Med. Mschr., 14, 25.

Szentagothai, J. (1948). J. Neurophysiol., 11, 445. 